\title{
Gene expression underlying enhanced, steroid-dependent auditory sensitivity of hair cell epithelium in a vocal fish
}

\author{
Daniel J. Fergus ${ }^{1,2}, \mathrm{Ni}$ Y. Feng ${ }^{1}$ and Andrew H. Bass ${ }^{1 *}$
}

\begin{abstract}
Background: Successful animal communication depends on a receiver's ability to detect a sender's signal. Exemplars of adaptive sender-receiver coupling include acoustic communication, often important in the context of seasonal reproduction. During the reproductive summer season, both male and female midshipman fish (Porichthys notatus) exhibit similar increases in the steroid-dependent frequency sensitivity of the saccule, the main auditory division of the inner ear. This form of auditory plasticity enhances detection of the higher frequency components of the multi-harmonic, long-duration advertisement calls produced repetitively by males during summer nights of peak vocal and spawning activity. The molecular basis of this seasonal auditory plasticity has not been fully resolved. Here, we utilize an unbiased transcriptomic RNA sequencing approach to identify differentially expressed transcripts within the saccule's hair cell epithelium of reproductive summer and non-reproductive winter fish.

Results: We assembled 74,027 unique transcripts from our saccular epithelial sequence reads. Of these, $6.4 \%$ and $3.0 \%$ were upregulated in the reproductive and non-reproductive saccular epithelium, respectively. Gene ontology (GO) term enrichment analyses of the differentially expressed transcripts showed that the reproductive saccular epithelium was transcriptionally, translationally, and metabolically more active than the non-reproductive epithelium. Furthermore, the expression of a specific suite of candidate genes, including ion channels and components of steroid-signaling pathways, was upregulated in the reproductive compared to the non-reproductive saccular epithelium. We found reported auditory functions for 14 candidate genes upregulated in the reproductive midshipman saccular epithelium, 8 of which are enriched in mouse hair cells, validating their hair cell-specific functions across vertebrates.

Conclusions: We identified a suite of differentially expressed genes belonging to neurotransmission and steroid-signaling pathways, consistent with previous work showing the importance of these characters in regulating hair cell auditory sensitivity in midshipman fish and, more broadly, vertebrates. The results were also consistent with auditory hair cells being generally more physiologically active when animals are in a reproductive state, a time of enhanced sensory-motor coupling between the auditory periphery and the upper harmonics of vocalizations. Together with several new candidate genes, our results identify discrete patterns of gene expression linked to frequency- and steroid-dependent plasticity of hair cell auditory sensitivity.
\end{abstract}

Keywords: Hearing, Hair cells, Saccule, Transcriptome, Frequency sensitivity, lon channels, Steroid hormones

\footnotetext{
* Correspondence: ahb3@cornell.edu

${ }^{1}$ Department of Neurobiology and Behavior, Cornell University, Ithaca, NY 14853, USA

Full list of author information is available at the end of the article
}

\section{Biomed Central}

(c) 2015 Fergus et al. Open Access This article is distributed under the terms of the Creative Commons Attribution 4.0 International License (http://creativecommons.org/licenses/by/4.0/), which permits unrestricted use, distribution, and reproduction in any medium, provided you give appropriate credit to the original author(s) and the source, provide a link to the Creative Commons license, and indicate if changes were made. The Creative Commons Public Domain Dedication waiver (http://creativecommons.org/publicdomain/zero/1.0/) applies to the data made available in this article, unless otherwise stated. 


\section{Background}

Understanding how genes are regulated within neural networks to produce and modify behavior is a major goal in neuroscience and behavioral genetics. One strategy for achieving this objective is to use model systems for identifying changing patterns of gene expression under different behavioral states. Exemplars include circadian rhythms in flies and mice e.g., [1-4], alternative foraging and aggressive behavioral states of honey bees e.g., $[5,6]$, and vocalacoustic systems of songbirds e.g., [7]. Here, we used transcriptome analyses to investigate the genetic underpinnings of reproductive state- and steroid-dependent plasticity in auditory sensitivity of a teleost fish, the midshipman.

Midshipman hearing is an excellent model of neural plasticity for several reasons. First, midshipman exhibit reproductive state-dependent behavioral responses to playback of advertisement calls [8]. Second, these behavioral changes are paralleled by concurrent changes in peripheral auditory sensitivity, both at the level of hair cells and eighth nerve, ganglion cell afferents, especially for the advertisement call's upper harmonics [9-11]. Third, auditory hair cell plasticity can be explained, in part, by changes in the abundance of ion channels that underlie frequency tuning [12]. Fourth, reproductive state-dependent variation in eighth nerve encoding of frequency is steroiddependent [13], providing a model for steroid-sensitive hearing variation in humans, in which age-related auditory deficits in post-menopausal woman can be ameliorated with estrogen therapy [14]. Fifth, the ease of collecting and housing midshipman fish in captivity facilitates downstream testing of identified candidate genes in a wild population of vertebrates.

To date, we have successfully employed hypothesisdriven approaches to identify neuro-molecular mechanisms of seasonal variation in peripheral auditory function, namely in the hair cell epithelium and eighth nerve afferents of the saccule, the main auditory division of the inner ear in many teleost fish including midshipman $[8,15]$. This has included examining the function, location, and abundance of ion channels and steroid receptors in the auditory periphery $[12,13,16,17]$. Neurophysiological studies show that either estrogen or testosterone can transform the frequency sensitivity of the saccular afferents of nonreproductive animals to that of summer animals [13]. Aromatase (estrogen synthase) and estrogen receptors are expressed within ganglion cells and the hair cell saccular epithelium, respectively [16, 17], indicating that steroids can act directly within the peripheral auditory system. Neurophysiology combined with pharmacology, quantitative reverse-transcriptase PCR, and immunohistochemistry also indicates that large-conductance potassium (BK) channel expression in saccular hair cells plays a key role in regulating the observed seasonal plasticity in auditory sensitivity [12].
Though our hypothesis-driven approach has been fruitful, it provides a limited view of the cascade of events underlying steroid-dependent, seasonal auditory plasticity. Advances in next-generation sequencing and high-throughput analyses can provide a global view of gene expression. Here, we use transcriptome sequencing to uncover seasonal and reproductive state-dependent differences in transcript abundances within the auditory saccular epithelium. We identified a suite of candidate genes and pathways with known auditory function in midshipman fish, and vertebrates in general, that likely underlie seasonal and reproductive state-dependent variation in hearing. The results have been reported, in part, in abstract form [18].

\section{Results and discussion}

\section{Transcriptome characterization}

Midshipman have two male reproductive morphs; we used type I males here because they are the most abundant during collections and have the most dynamic vocal repertoire [19, 20]. Given the lack of sex [11] and male morph [21] differences in auditory hair cell physiology, the use of only type I males should not impact our results. Here, we focus on the subset of transcriptome sequences from the auditory saccular epithelium (SE) of reproductive (summer) and non-reproductive (winter) type I males that were previously used for physiological examination of auditory sensitivity [11].

We sequenced and annotated transcriptome libraries produced from the SE, along with libraries derived from the vocal motor nuclei (VMN) and the hindbrain region surrounding the VMN. The VMN and hindbrain samples were used for a comprehensive companion study that identified daily and seasonal variation in gene expression patterns in the VMN, the final node of the vocal control network that sends a command signal to the vocal muscles [22]. In that study, the RNA-seq determined expression patterns of 28 genes were validated using quantitative PCR, showing a strong correlation between the two measures of transcript abundance [22].

We obtained approximately 200 million total paired-end reads; over 20 million reads were produced from each pooled SE sample (Table 1). Using the Trinity software package (version r2013-02-15 [23, 24]) we assembled all the reads together into a final set of 83,967 unique transcripts (isoforms) after filtering for quality of reads and of assembled transcripts [22]. The final transcriptome

Table 1 Number of saccular epithelium reads by reproductive state before and after quality filtering

\begin{tabular}{lll}
\hline Reproductive state & Raw forward reads & Filtered paired-end reads \\
\hline Reproductive & 23112842 & 21401113 \\
Non-reproductive & 24208024 & 21617526 \\
\hline
\end{tabular}


assembly, reported in our companion study [22], had a mean length of $1713.57 \pm 1585.21 \mathrm{bp}(\mathrm{N} 50=2647)$ with 40,656 genes (components) across brain and SE samples. The assembled transcriptome is available on the NCBI Transcriptome Shotgun Assembly and Sequence Read Archive databases under BioProject accession number PRJNA269550. Using Blast2GO, we found significant annotation hits for 74,000 (88 \%) of our assembled transcripts, with most top blast hits being to teleost fish (see [22]). Mapping individual reads back to the assembled transcriptome and comparing FPKM (fragments per kilobase per million reads) values showed that most transcripts were expressed $(\mathrm{FPKM}>0)$ in all examined tissue types, though the SE possessed the largest number of tissue-specific transcripts that had no expression $(\mathrm{FPKM}=0)$ in either VMN or hindbrain (Fig. 1). This likely reflects the different developmental trajectory and cell types of the inner ear (placode) relative to the VMN and hindbrain (rhombomeres) (see [25]).

\section{Reproductive-state specific expression}

We first examined top expressed transcripts and pathways in reproductive and non-reproductive SE, regardless of differential expression. For transcripts with significant Blast2GO annotations, 6 of the top 10 most highly expressed transcript annotations were common to both reproductive and non-reproductive SE although they varied in expression levels (Table 2). Four of the top 10 most abundant transcripts in the reproductive SE encode ribosomal proteins, while only one of those in the non-reproductive SE encodes a ribosomal protein.
The translationally controlled tumor protein $(t p t 1)$, which functions to prevent cell death [26, 27], was among the most abundant transcripts in the reproductive SE and may play a role in the increased hair cell numbers previously reported in the reproductive SE [28].

We also identified the top 10 KEGG (Kyoto Encyclopedia of Genes and Genomes) pathways based on the number of annotated transcripts that mapped to each pathway for reproductive and non-reproductive SE (Table 3). It is noteworthy that each of the top KEGG pathways for the reproductive SE are represented by far more transcripts, on average, than the top non-reproductive KEGG pathways. This may have resulted, in part, from completely "turning off" more complex pathways in the non-reproductive SE and/or decreased transcription of certain genes. For example, among the highly represented KEGG pathways in reproductive SE were processes involved in cellular respiration, including oxidative phosphorylation, glycolysis, TCA cycle, and pyruvate metabolism. While cellular respiration unquestionably occurs in the SE throughout the entire year to support year-round hearing $[10,11]$, the reduced transcript representation of these cellular respiration KEGG pathways in non-reproductive SE suggests a higher energetic demand in the reproductive state that corresponds to greater SE auditory sensitivity $[9,11]$.

We examined the differential regulation of gene expression in the SE across reproductive states by performing differential expression analyses of genes (components) and transcripts (isoforms). We compared transcript abundances across all the SE, VMN and surrounding hindbrain tissue groups using a false discovery

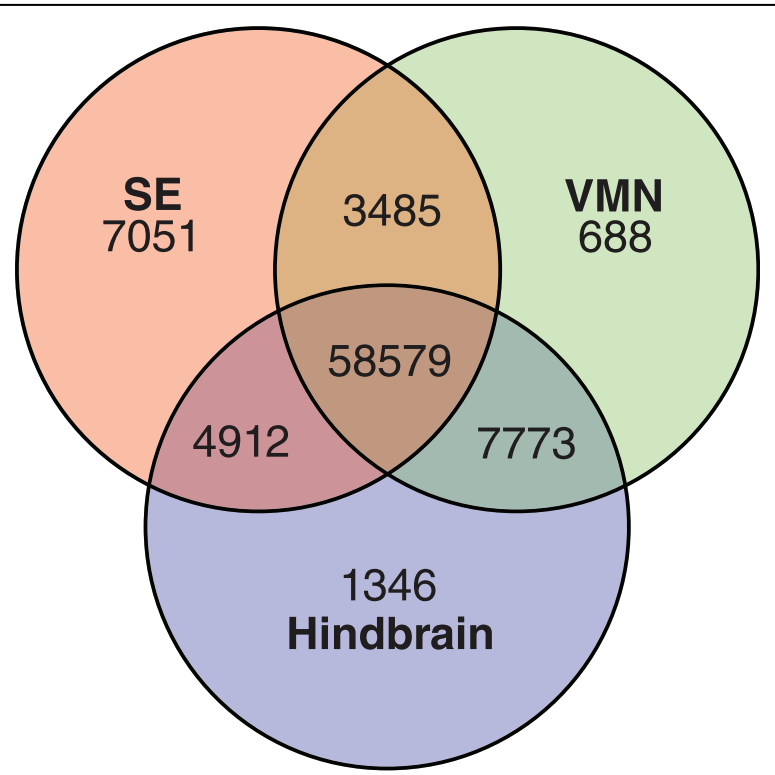

Fig. 1 Common and unique transcripts among tissues. The Venn diagram illustrates the common and unique transcripts across saccular hair cell epithelium (SE), VMN, and hindbrain tissues [22]. Most transcripts were shared among all tissues, while SE had the largest number of unique transcripts, consistent with their unique (epithelial) tissue type 
Table 2 Top 10 most highly expressed annotated saccular epithelium transcripts

\begin{tabular}{|c|c|c|c|c|c|}
\hline$\overline{\text { Reproductive }}$ & Sequence ID & FPKM & Non-reproductive & Sequence ID & FPKM \\
\hline Myoglobin & comp194478_c0_seq1 & 25048 & $\begin{array}{l}\text { Serine threonine-protein kinase samkc-like } \\
\text { isoform } \times 2\end{array}$ & comp203376_c0_seq1 & 36792 \\
\hline $\begin{array}{l}\text { Serine threonine-protein kinase samkc-like } \\
\text { isoform } \times 2\end{array}$ & comp203376_c0_seq1 & 24585 & Myoglobin & comp194478_c0_seq1 & 29279 \\
\hline Mucin-22-like & comp203306_c0_seq3 & 9686 & Hemoglobin subunit beta-like & comp194456_c0_seq1 & 21830 \\
\hline Male-specific protein & comp237277_c0_seq1 & 8890 & Mucin-22-like & comp203306_c0_seq3 & 15462 \\
\hline Inner ear-specific collagen-like & comp221658_c0_seq1 & 8616 & Inner ear-specific collagen-like & comp221658_c0_seq1 & 13183 \\
\hline Translationally controlled tumor protein & comp126388_c0_seq1 & 7895 & Male-specific protein & comp237277_c0_seq1 & 9766 \\
\hline Ribosomal protein 112 & comp172912_c0_seq1 & 6996 & Matrilin-4 isoform 1 & comp210154_c1_seq1 & 8186 \\
\hline $40 S$ ribosomal protein $s 27$ & comp194407_c0_seq1 & 6806 & 405 ribosomal protein $s 8$ & comp126398_c0_seq1 & 7949 \\
\hline 405 ribosomal protein s8 & comp126398_c0_seq1 & 6681 & $\beta$-actin & comp126442_c0_seq1 & 7226 \\
\hline 60 s ribosomal protein 132 & comp126412_c0_seq1 & 6011 & a-type globin & comp221632_c0_seq3 & 6652 \\
\hline
\end{tabular}

rate (FDR) threshold of 0.001 and a minimum four-fold differential abundance cutoff (Figs. 2, 3). Based on pairwise comparisons of differential transcript abundances, the samples were more similar between time points for a single tissue type than between tissues. Furthermore, in all tissue types, transcript isoforms showed greater differential expression than gene components across time points, suggesting that variation across seasons was determined more by differential splicing than by turning the expression of a given gene on or off. The substantial expression change parallels the observed neurophysiological change in SE auditory sensitivity between reproductive and non-reproductive individuals $[9,11]$. Furthermore, the SE showed higher seasonal differentiation in both gene and transcript expression relative to either VMN or the surrounding hindbrain (Figs. 2, 3), suggesting that at the level of hair cells and motoneurons, seasonal variation in hearing sensitivity requires greater transcriptional changes than the vocal motor system.
Focusing specifically on transcripts differentially expressed between reproductive and non-reproductive SE, we identified 4713 and 2221 upregulated in reproductive and non-reproductive SE, respectively. Performing the same analysis with genes rather than transcripts produced 878 and 24 genes upregulated in reproductive and non-reproductive SE, respectively. We employed a Fisher's Test for gene ontology (GO) term enrichment with Blast2GO to determine whether there was an overrepresentation of any classes of functionally similar transcripts that were differentially regulated across seasons. Many GO terms were over-represented among transcripts upregulated in reproductive SE, while none were over-represented among transcripts upregulated in non-reproductive SE (Table 4). The most significantly enriched GO terms were primarily related to translation (e.g., ribosomal proteins) and cellular respiration, supporting results of the overall most abundant transcripts (Table 2) and most supported KEGG pathways (Table 3) discussed above.

Table 3 Top 10 KEGG pathways in the saccular epithelium by number of transcripts

\begin{tabular}{|c|c|c|c|c|c|}
\hline Reproductive & KEGG ID & Transcripts & Non-reproductive & KEGG ID & Transcripts \\
\hline Purine metabolism & map00230 & 107 & Purine metabolism & map00230 & 66 \\
\hline Oxidative phosphorylation & map00190 & 50 & Pyrimidine metabolism & map00240 & 27 \\
\hline Glycolysis/Gluconeogenesis & map00010 & 45 & Thiamine metabolism & map00730 & 13 \\
\hline Pyrimidine metabolism & map00240 & 38 & Phosphatidylinositol signaling system & map04070 & 12 \\
\hline Citrate cycle (TCA cycle) & map00020 & 30 & Aminoacyl-tRNA biosynthesis & map00970 & 10 \\
\hline Carbon fixation pathways in prokaryotes & map00710 & 28 & Lysine degradation & map00310 & 9 \\
\hline Pyruvate metabolism & map00620 & 25 & Glycerophospholipid metabolism & map00564 & 7 \\
\hline Glutathione metabolism & map00480 & 25 & Inositol phosphate metabolism & map00562 & 7 \\
\hline Carbon fixation in photosynthetic organisms & map00710 & 24 & Various types of $\mathrm{N}$-glycan biosynthesis & map00513 & 6 \\
\hline Glycine, serine and threonine metabolism & map00260 & 24 & One carbon pool by folate & map00670 & 6 \\
\hline
\end{tabular}




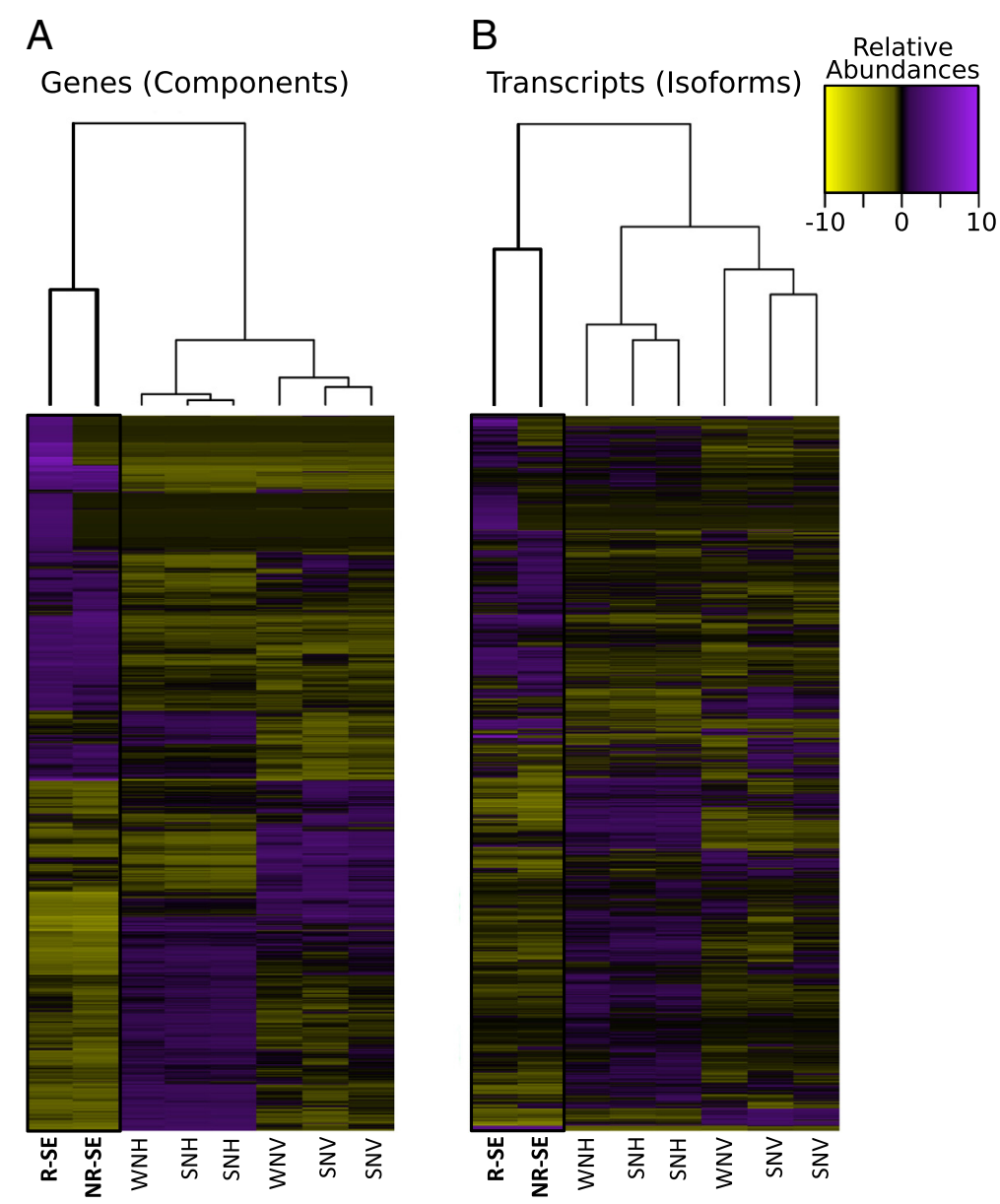

Fig. 2 Heatmaps of tissue specific expression. Heatmaps showing normalized expression of differentially expressed (a) genes and (b) transcripts among saccular epithelium (SE) and brain tissues. Differential expression is based on a minimum 4-fold differential variation among tissues with a maximum false discovery rate (FDR) of 0.001 . While samples grouped by tissue type, the SE showed strong differentiation by season. The SE columns, the focus of this study, are highlighted by a black box. Abbreviations: R-SE, reproductive saccular epithelium; NR-SE, non-reproductive saccular epithelium; WNH, winter night hindbrain; SMH, summer morning hindbrain; SNH, summer night hindbrain; WNV, winter night VMN (vocal motor nucleus); SMV, summer morning VMN; SNV, summer night VMN

As a control for our differential analyses in the SE, we performed comparable seasonal analyses for sample groups of the hindbrain region surrounding VMN that were used in our companion study of the vocal motor system [22]. Using the same differential expression criteria, we found that 2157 and 1984 transcripts were upregulated in reproductive and non-reproductive hindbrains, respectively. There were 3 and 12 genes upregulated in the reproductive and non-reproductive hindbrain, respectively. Among the differentially expressed transcripts, only a single GO term, "protein-binding", was significantly enriched in the reproductive hindbrain. Thus, transcript abundance and GO term enrichment differences did not reflect ubiquitous seasonal or reproductive state variation, but rather SE-specific transcriptional regulation.

Taken together, the results identified broad changes in gene expression between reproductive and nonreproductive states of high and low saccular activity, respectively. The substantially greater number of transcripts and genes upregulated in reproductive relative to nonreproductive samples suggested a general increase in transcriptional activity. The absence of this pattern in the hindbrain region surrounding VMN indicated that the increased transcription in the reproductive state is an SEspecific phenomenon. Furthermore, the enriched GO terms (Table 4) of the differentially expressed transcripts pointed to a much greater translational and metabolic activity in the reproductive SE than the non-reproductive $\mathrm{SE}$, consistent with the most abundant annotations and KEGG pathways reported in Tables 2 and 3. We thus conclude that the SE is transcriptionally, translationally, and metabolically more active in reproductive than non-reproductive individuals.

\section{Candidate gene identification}

The overarching goal of this study was to identify candidate genes that may influence seasonal variation 
A

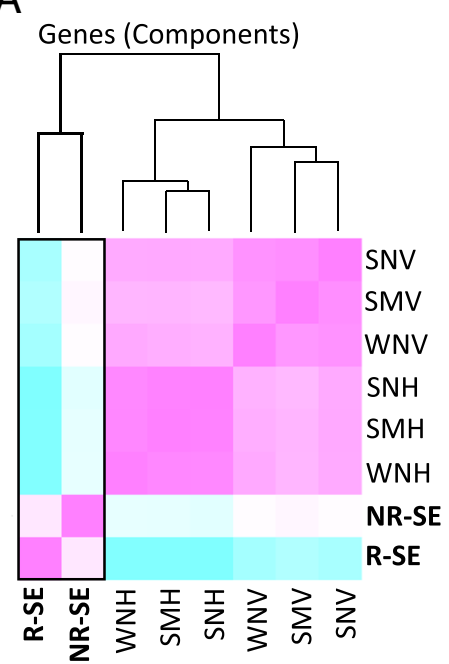

$B$

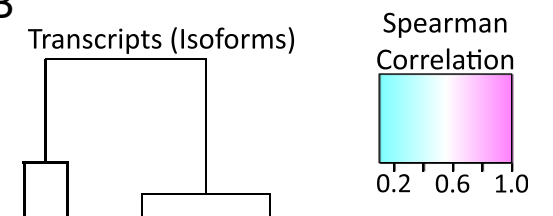

Fig. 3 Spearman's correlation of gene and transcript expression. Sample relationships based on (a) gene and (b) transcript expression were scaled to a color gradient based on Spearman's correlations among tissue groups. As in Fig. 2, the saccular epithelium (SE) tissues grouped together, but still showed strong differentiation between seasons. This difference is much more apparent at the level of the transcripts than the genes. The SE columns, the focus of this study, are highlighted by a black box. Abbreviations are as in Fig. 2

in auditory frequency sensitivity. While the analyses above suggested broad changes in activity and metabolism across reproductive states, we hypothesized that the known physiological changes in auditory sensitivity likely depend predominantly on changes in a smaller subset of genes important for neural function and neuronal excitability e.g., [12]. While our approach here may have missed variation of low abundance transcripts and small magnitude expression differences, we identified numerous candidate genes for further study. Furthermore, many of the identified candidates have previously been implicated in vertebrate auditory function (Table 5, [29]).

Candidate genes upregulated in the reproductive SE that support prior studies of midshipman auditory function included estrogen related receptors (ERR), neuronal acetylcholine receptor subunit $\alpha-9-i i$, insulin gene enhancer protein isl-1, and diaphanous 1. The ability of estrogen to enhance auditory sensitivity in the midshipman SE is well established [13], and estrogen receptors have been localized to the SE [16]. ERRs exert estrogenlike actions by activating genes regulated by estrogenresponse elements in the absence of the ligand [30-32], and mutations of ERRs have been implicated in hearing impairments in mice and humans ([33-35]). Increased ERR expression may serve as a supplemental or alternative means to estrogenic actions in regulating genes important for maintaining high-frequency auditory sensitivity.

The neuronal acetylcholine receptor subunit $\alpha-9-i i$, also upregulated in reproductive SE, is expressed in hindbrain neurons that directly innervate the inner ear (i.e., auditory efferents) of teleosts [36] that have been shown in midshipman to be part of a vocal corollary discharge pathway linking the hindbrain vocal pattern generator to the auditory saccule [37]. In mammals, which lack the $\alpha-9$-ii receptor, acetylcholine receptor subunits $\alpha-9$ and $\alpha-10$ are highly expressed in auditory hair cells [29] and form heterotetramers at the auditory efferent synapses with cochlear hair cells (see [38]), likely regulating the dynamic range of hearing and protecting against environmental or self-generated acoustic trauma. In midshipman, acetylcholine receptor subunit $\alpha$-9-ii may either be involved in setting the auditory sensitivity thresholds across frequencies or help protect the SE in the potentially more acoustically active tidal environment inhabited during the reproductive summer compared to the deep water environment inhabited during the non-reproductive winter. Ambient sound levels during the summer reproductive period may be higher due to increased vocal activity especially in and close to nests $[19,20,39]$, as well as increased noise levels in the shallow water environment e.g., [39, 40] where midshipman build nests and spawn [19, 20].

The insulin gene enhancer protein isl-1 is an interesting candidate gene based on recent work by Huang et al. [41], which showed that over-expression of Isl-1 in mouse hair cells prevented age-related and noise-induced hearing loss resulting from hair cell apoptosis or neural degeneration. Another candidate gene with similar implications for auditory sensitivity and hair cell proliferation is diaphanous 1 whose mutation contributes to progressive hearing loss [42]. Products of the diaphanous genes contribute to cytoskeletal function including establishing cell polarity and shape [43-45]. Both of these candidate genes could contribute to the increased auditory sensitivity at high 
Table 4 Enriched GO terms among upregulated transcripts in reproductive saccular epithelium

\begin{tabular}{|c|c|c|c|}
\hline & GO-ID & GO term & $p$ \\
\hline \multirow[t]{20}{*}{ Biological process } & GO:0000184 & Nuclear-transcribed mRNA catabolic process, nonsense-mediated decay & $1.2 \mathrm{E}-14$ \\
\hline & GO:0006614 & SRP-dependent cotranslational protein targeting to membrane & $6.6 \mathrm{E}-14$ \\
\hline & GO:0006415 & Translational termination & $2.4 \mathrm{E}-13$ \\
\hline & GO:0015986 & ATP synthesis coupled proton transport & $3.9 \mathrm{E}-12$ \\
\hline & GO:0019083 & Viral transcription & $6.7 \mathrm{E}-11$ \\
\hline & GO:0006446 & Regulation of translational initiation & $9.7 \mathrm{E}-11$ \\
\hline & GO:0006120 & Mitochondrial electron transport, NADH to ubiquinone & $8.6 \mathrm{E}-10$ \\
\hline & GO:0006744 & Ubiquinone biosynthetic process & $2.1 \mathrm{E}-09$ \\
\hline & GO:0006123 & Mitochondrial electron transport, cytochrome c to oxygen & $2.1 \mathrm{E}-09$ \\
\hline & GO:0006096 & Glycolysis & $1.2 \mathrm{E}-08$ \\
\hline & GO:0006457 & Protein folding & 1.7E-08 \\
\hline & GO:0006094 & Gluconeogenesis & $2.6 \mathrm{E}-08$ \\
\hline & GO:0000028 & Ribosomal small subunit assembly & $2.0 \mathrm{E}-07$ \\
\hline & GO:0031101 & Fin regeneration & $2.1 \mathrm{E}-07$ \\
\hline & GO:0006448 & Regulation of translational elongation & $2.1 \mathrm{E}-07$ \\
\hline & GO:0019643 & Reductive tricarboxylic acid cycle & $1.6 \mathrm{E}-06$ \\
\hline & GO:0015976 & Carbon utilization & $3.0 \mathrm{E}-05$ \\
\hline & GO:0030036 & Actin cytoskeleton organization & $3.4 \mathrm{E}-05$ \\
\hline & GO:0006364 & rRNA processing & $3.9 \mathrm{E}-05$ \\
\hline & GO:0005980 & Glycogen catabolic process & $6.2 \mathrm{E}-05$ \\
\hline \multirow[t]{11}{*}{ Molecular function } & GO:0003735 & Structural constituent of ribosome & $4.6 \mathrm{E}-37$ \\
\hline & GO:0008137 & NADH dehydrogenase (ubiquinone) activity & $6.2 \mathrm{E}-11$ \\
\hline & GO:0003743 & Translation initiation factor activity & $1.1 \mathrm{E}-09$ \\
\hline & GO:0004129 & Cytochrome-c oxidase activity & $2.5 \mathrm{E}-09$ \\
\hline & GO:0051082 & Unfolded protein binding & $9.4 \mathrm{E}-08$ \\
\hline & GO:0046933 & Proton-transporting ATP synthase activity, rotational mechanism & 5.7E-07 \\
\hline & GO:0003746 & Translation elongation factor activity & $2.4 \mathrm{E}-06$ \\
\hline & GO:0019843 & rRNA binding & $1.8 \mathrm{E}-05$ \\
\hline & GO:0004365 & GAPDH (NAD+) (phosphorylating) activity & $6.7 \mathrm{E}-05$ \\
\hline & GO:0072542 & Protein phosphatase activator activity & $8.5 \mathrm{E}-05$ \\
\hline & GO:0016936 & Galactoside binding & $9.9 \mathrm{E}-05$ \\
\hline \multirow[t]{12}{*}{ Cellular component } & GO:0022627 & Cytosolic small ribosomal subunit & $1.5 \mathrm{E}-22$ \\
\hline & GO:0022625 & Cytosolic large ribosomal subunit & $1.3 \mathrm{E}-12$ \\
\hline & GO:0005852 & Eukaryotic translation initiation factor 3 complex & $1.6 \mathrm{E}-10$ \\
\hline & GO:0045277 & Respiratory chain complex IV & $2.1 \mathrm{E}-09$ \\
\hline & GO:0045095 & Keratin filament & $6.0 \mathrm{E}-09$ \\
\hline & GO:0005747 & Mitochondrial respiratory chain complex I & $2.0 \mathrm{E}-08$ \\
\hline & GO:0045261 & Proton-transporting ATP synthase complex, catalytic core F(1) & $3.5 \mathrm{E}-07$ \\
\hline & GO:0042470 & Melanosome & 4.3E-05 \\
\hline & GO:0005730 & Nucleolus & 5.7E-05 \\
\hline & GO:0005861 & Troponin complex & $5.9 \mathrm{E}-05$ \\
\hline & GO:0005832 & Chaperonin-containing T-complex & $6.2 \mathrm{E}-05$ \\
\hline & GO:0000276 & Mitochondrial $\mathrm{H}^{+}$-transporting ATP synthase complex, coupling factor $\mathrm{F}(\mathrm{O})$ & $6.2 \mathrm{E}-05$ \\
\hline
\end{tabular}


Table 5 Differentially expressed candidate genes in the saccular epithelium with reported auditory functions and comparison to mouse hair cell expression

\begin{tabular}{|c|c|c|c|}
\hline Top blast hit description & Season & Citation & $\begin{array}{l}\text { Mouse } \\
\mathrm{HC}\end{array}$ \\
\hline Vesicular glutamate transporter 3 & $\mathrm{R}$ & {$[81]$} & + \\
\hline Estrogen-related receptor $\beta$ type 1 & $\mathrm{R}$ & [33] & $=$ \\
\hline Thyroid hormone receptor a & $R / N R$ & [82] & $=$ \\
\hline Connexin 43 (Gap junction a-1) & $\mathrm{R} / \mathrm{NR}$ & {$[83]$} & - \\
\hline $\begin{array}{l}\text { Neuronal acetylcholine receptor } \\
\text { subunit a-9-ii }\end{array}$ & $\mathrm{R}$ & [36] & + \\
\hline $\begin{array}{l}\text { Potassium voltage-gated channel } \\
\text { subfamily a member } 10\end{array}$ & $\mathrm{R}$ & {$[84]$} & + \\
\hline Sodium channel protein type 8 subunit a & $\mathrm{R}$ & {$[85]$} & $=$ \\
\hline Diaphanous homolog 1-like isoform $\times 2$ & $\mathrm{R}$ & [42] & $={ }^{\mathrm{b}}$ \\
\hline Insulin gene enhancer protein isl-1 & $\mathrm{R}$ & [41] & + \\
\hline Estrogen-related receptor $\gamma$ & $\mathrm{R} / \mathrm{NR}$ & [35] & + \\
\hline $\begin{array}{l}\text { Calcium-activated potassium channel } \\
\text { subunit a-1 (BK) }\end{array}$ & $\mathrm{R}$ & [12] & $=$ \\
\hline Connexin 30 (Gap junction $\beta-6$ ) & $\mathrm{R} / \mathrm{NR}$ & [65] & - \\
\hline $\begin{array}{l}\text { Trimeric intracellular cation channel } \\
\text { type a }\end{array}$ & $\mathrm{R}$ & {$[86]$} & + \\
\hline $\begin{array}{l}\text { Voltage-dependent calcium channel } \\
\text { subunit a-2 } \delta-3\end{array}$ & $\mathrm{R} / \mathrm{NR}$ & {$[87]$} & $=$ \\
\hline
\end{tabular}

These differential expressed transcripts have previously been implicated in peripheral auditory function. Shown are the top Blast hit descriptions, the season during which transcript abundance was highest (R: reproductive; NR: non-reproductive), and a citation for the auditory role of that gene. In cases where both seasons are listed, different isoforms were upregulated in both seasons. The "Mouse HC" column represents comparisons of the average normalized mRNA transcript abundances of FAC sorted hair cells to that of surrounding cells from embryonic day 16 and postnatal days 0,4 , and 7 mouse cochlea [29]. We indicated whether expression in hair cells was not substantially different $(=),>2$ fold higher $(+)$, or $>2$ fold lower $(-)$, than in the surrounding cells of the cochlea. The first 8 transcripts in this table were further supported by an examination of postnatal day 1 mouse organ of corti [88]. ${ }^{a}$ Neuronal acetylcholine receptor subunit a-9-ii (Chrna9-ii) is not present in mammals; comparable genes Chrna9 and Chrna10 transcripts are both more abundant in hair cells than surrounding cells.

${ }^{\text {b}}$ Diaphanous homolog 1 is not reported in [29] and this comparison is based only on [88]

frequencies as well as the reported increase in hair cell number in the reproductive SE [28].

There was little overlap between the candidate genes identified in this study and those that are activated during regeneration following acoustic trauma in zebrafish [46]. Given the seasonal variation in hair cell abundance in midshipman [28], we might expect some overlap among these processes. The presence of some similar classes of genes, such as myosin genes and orthologous nuclear receptors, in both analyses may stem from the regeneration of new hair cells in both model systems. However, the amount of hair cell regeneration and the underlying mechanisms may vary substantially between traumainduced and naturally occurring seasonal regeneration.

There were additional upregulated genes in the reproductive SE that have not previously been directly implicated in auditory function but still serve as important candidates for consideration and future validation. These included steroid related genes such as the transcript hydroxysteroid 11- $\beta$-dehydrogenase 1, which converts cortisol to the inactive metabolite cortisone (see [47, 48]), as well as transcripts encoding glucocorticoid receptors (Additional file 1). Though cortisol has not been directly implicated in auditory function, there is extensive evidence for a role of glucocorticoids in hearing [49-53] and in lateral line hair cell regeneration [54]. In teleost fish, hydroxysteroid 11- $\beta$-dehydrogenase also converts 11beta-hydroxytestosterone to 11-ketotestosterone (11KT), a non-aromatizable androgen detectable only in reproductive type I males $[55,56]$. While $11 \mathrm{KT}$ has an effect on vocalization $[57,58]$, its role in auditory physiology has not been examined. The auditory sensitivity of primary afferents innervating the SE of non-reproductive fish can be shifted to that of reproductive fish by testosterone [13], which may act via local conversion to estrogen by aromatase in the ganglion cells [17]. This mechanism would compete with the conversion of testosterone to $11 \mathrm{KT}$, which could only act by direct activation of an androgen receptor (AR). In situ hybridization shows AR $\beta$ mRNA in the region directly adjacent to saccular hair cells [59]. Transcripts of both AR $\alpha$ and AR $\beta$ are detectable by qPCR at approximately equal abundances in the SE of all reproductive morphs (D. Fergus and A. Bass, unpublished observations), but seasonal variation in expression of either AR has not been directly tested and was not detected in our results here. Many genes like hydroxysteroid 11- $\beta$ dehydrogenase 1 could be critical for the physiological changes across reproductive states in midshipman SE, but have not been studied in the context of auditory plasticity prior to this transcriptome differential expression analysis.

Neurophysiology shows that ion channels and steroid hormones are critical to the increased auditory sensitivity in reproductive midshipman fish [12,13] (see also [60] for qPCR of steroid receptors in SE of a cichlid fish). To take a more targeted approach for our candidate gene search, we identified 1547 ion channel transcripts and 361 steroid-related transcripts within our entire assembled transcriptome and performed differential expression analyses with each of these transcript subsets. This approach reduced the number of pairwise comparisons, allowing us to potentially increase our sensitivity to detect differentially expressed transcripts with functional importance. As with the whole transcriptome, substantially more steroid-related (Additional file 1) and ion channel (Additional file 2) transcripts were upregulated in reproductive compared to non-reproductive SE.

Among the ion channels upregulated in reproductive SE were large conductance, calcium-activated potassium (BK) channel transcripts (Table 5, Additional file 2) that have been localized to saccular hair cells in midshipman, 
shown to vary in abundance across seasons and to regulate auditory sensitivity in midshipman adults and zebrafish larvae [12, 61]. Numerous other potassium channel transcripts were also differentially regulated across seasons (Additional file 2), which may be necessary for the fine-tuning of auditory thresholds, as demonstrated in other vertebrates [62] and suggested by our auditory physiology studies of the SE [12].

One steroid-related and several channel transcripts identified as upregulated in reproductive SE were also upregulated in the hindbrain and/or VMN of reproductive state fish (see companion RNA-seq study [22]) (Table 6). The common reproductive-state dependent regulation of these transcripts may support broadly shared motor and sensory functions. Two channel transcripts that showed reproductive upregulation in the VMN and SE, but not the hindbrain, connexin 30 (Cx30, gap junction $\beta-6)$ and calcium-activated potassium channel subunit $\alpha-1$ (BK) (Table 6), are particularly interesting in light of our previous neurophysiological examinations of midshipman vocal and auditory systems. Connexins are gap junction proteins that contribute to electrical coupling between cells and are abundant in glia [63]. Though not yet tested, $C \times 30$ might support the known electrical coupling between VMN motoneurons and, in turn, the extreme, population level synchronicity observed for VMN [64]. In the inner ear, Cx30 occurs between supporting cells in the cochlear hair cell epithelium; mice lacking Cx30 show severe hearing loss [65]. There is evidence for gap junctions between supporting cells of the SE and possibly between hair cells and supporting cells in toadfish from the same family as midshipman [66]. BK channels, as noted earlier, are more abundant in the SE of reproductive midshipman, playing a

Table 6 Channel and steroid-related candidate genes upregulated in the vocal system

\begin{tabular}{|c|c|}
\hline Reproductive & Vocal Upregulation \\
\hline Cholesterol 25-hydroxylase protein member 1 & H/NMN \\
\hline Connexin 43 (Gap junction a-1) & HNMN \\
\hline $\begin{array}{l}\text { Voltage-gated potassium channel subfamily } \\
\text { c member } 4\end{array}$ & HNMN \\
\hline Sodium channel protein type 8 subunit a & H/NMN \\
\hline Two pore calcium channel protein 1 & HNMN \\
\hline Connexin 30 (Gap junction $\beta-6$ ) & VMN \\
\hline $\begin{array}{l}\text { Calcium-activated potassium channel } \\
\text { subunit a-1 (BK) }\end{array}$ & Night VMN \\
\hline $\begin{array}{l}\text { Transient receptor potential cation channel } \\
\text { subfamily m member } 7\end{array}$ & VMN \\
\hline Anoctamin-10 & Night VMN \\
\hline \multicolumn{2}{|c|}{$\begin{array}{l}\text { Transcripts of these candidate genes, upregulated in reproductive SE, were } \\
\text { also more abundant in vocal regions of the CNS of reproductive type I males } \\
\text { [22]. Some transcripts were upregulated throughout the hindbrain and VMN } \\
\text { (H/VMN), some were upregulated only in the VMN (VMN), and others had } \\
\text { increased abundance restricted to VMN at night (Night VMN), the time of peak } \\
\text { vocal activity }\end{array}$} \\
\hline
\end{tabular}

prominent role in the sensitivity of SE hair cells to the full $\sim 100-400 \mathrm{~Hz}$ spectral range of their vocalizations [12]. BK channels may also contribute to high fidelity firing in VMN that codes for vocalization pulse repetition rates and fundamental frequencies of $\sim 100-110 \mathrm{~Hz}$ [20]. In support of this potential vocal function, recent studies demonstrate a role for BK channels in high fidelity firing $(\sim 50-100 \mathrm{~Hz})$ by Purkinje cell axons in the cerebellum [67]. The co-regulation of such genes in two highly divergent neural systems, one sensory and one motor, is compelling given the importance of sender-receiver/vocalauditory coupling in the acoustic communication system of the plainfin midshipman $[8,12,13,37,68]$.

\section{Conclusion}

We uncovered the molecular underpinnings of reproductive state-dependent variation of auditory sensitivity in midshipman fish. Our results suggested broad changes in transcriptional, translational, and metabolic activity occurring in the SE across reproductive states, with higher activity in the reproductive state. In addition to these broad changes, differential expression analyses identified a number of potential candidate genes underlying seasonal changes in auditory physiology. Some of these genes, such as potassium channels and steroid biogenesis enzymes, are highly consistent with our previous work, while others, like insulin gene enhancer protein isl-1, neuronal acetylcholine receptor $\alpha-9-i i$ and diaphanous 1 , are implicated in mammalian hair cell function and thus provide novel targets for future investigation in fish model systems.

We have previously shown that the magnitude of the seasonal change in hearing thresholds in the SE is significantly greater for encoding the higher frequency, upper harmonics of the male advertisement call ([11], also see [13]). Midshipman fish migrate from nest sites in the shallow intertidal zone to deep off shore sites during the non-reproductive winter season e.g. [56]. Despite the apparent attenuation of transcription, translation, and metabolic activity in the nonreproductive SE that we report here, the SE retains robust sensitivity to low frequency sound $(\leq 100 \mathrm{~Hz})[10-13,68]$. Such low frequency sensitivity in deep water sites has been proposed to be important for detection of the brief ( $200 \mathrm{msec})$, low frequency agonistic grunts of conspecifics that are produced all year long $[19,20]$ and the low frequency calls of marine mammals, both of which will have a greater transmission distance in deeper water (see $[39,69])$. Seasonal changes in hearing are not observed in a closely related species of toadfish that does not migrate to deeper waters during the non-reproductive season [70]. Perhaps the basal condition for toadfishes is high sensitivity across a wide range of frequencies and spectral peaks. In this case, selective pressure may have actively suppressed sensitivity to high frequencies while maintaining sensitivity for low frequencies in the non-reproductive winter SE of midshipman 
fish, rather than actively enhancing the higher frequency hearing in a summer reproductive fish. While this does not drastically change our questions regarding seasonal variation in auditory sensitivity, it can inform our thinking about how and why species like midshipman evolved the physiological and genetic mechanisms underlying frequency-dependent seasonal plasticity in hearing.

\section{Methods}

\section{Collection}

All fish used in this study were type I males. The SE were from reproductive males collected in summer and non-reproductive males collected in winters of 2009 and 2010 in California and Washington. These fish, previously used to examine seasonal variation in auditory sensitivity and steroid levels [11], were collected from nest sites, shipped back to Cornell University, and housed in artificial seawater aquaria maintained at $16{ }^{\circ} \mathrm{C}$ until they were used for neurophysiology and sacrificed to collect tissues. The ears were removed, dissected to isolate the SE from surrounding ear tissue, immediately frozen in liquid nitrogen, and stored at $-80{ }^{\circ} \mathrm{C}$ until being used for RNA isolation. All procedures used here were approved by Cornell University's Institutional Animal Care and Use Committee.

\section{Library construction}

The methods for library construction, sequencing, and transcriptome assembly here are the same as those used in our companion study of the vocal network [22]. Total RNA was isolated from the SE of 6 reproductive and 6 non-reproductive fish using the Trizol reagent (Invitrogen) following the manufacturer's standard protocol. The isolated total RNA was quantified using the Qubit RNA HS quantification kit (Invitrogen) and equal quantities of RNA from each ear were pooled by reproductive state. DNase I (Ambion) treatment was performed on each pool to remove contaminating DNA.

We constructed indexed, strand-specific cDNA libraries using the deoxyuridine triphosphate (dUTP)/uracil-DNA glycosylase (UDG) approach described by Zhong et al. [71]. Briefly, mRNA was purified from the total RNA using Dynabeads Oligo(dT) 25 (Life Technologies) and then fragmented to approximately $200 \mathrm{bp}$ with divalent cation buffer (SuperScript III buffer, Life Technologies). This fragmented mRNA was used to produce first-strand cDNA with dNTPs and SuperScript III enzyme. We then produced second-strand CDNA with dUTP substituted for dTTP, dA-tailed the double-stranded DNA fragments, and ligated Y-adapters created by annealing primers with both complimentary and non-complimentary regions.

After the Y-adapters had been ligated to the doublestranded DNA fragments, we purified and size selected them using AMPure beads (Beckman Coulter, Inc.). The uracil containing second-strand DNA was digested with uracil-DNA glycosylase. After digesting the secondstrand, the remaining first-strand cDNA had unique sequence tags on the $5^{\prime}$ and $3^{\prime}$ ends that served as priming sites for PCR primers containing indexes and epitopes necessary for Illumina sequencing. We used 14 cycles of PCR reactions to produce double-stranded cDNA fragments that were uniquely indexed for summer reproductive and winter non-reproductive SE. The resulting DNA was purified, verified by both gel electrophoresis and Agilent Bioanalyzer, which showed peaks between 251 and 257 bp. The DNA was quantified with the Qubit dsDNA HS quantification kit (Invitrogen), and combined in equal quantities (20 ng per pool) with the vocal motor nucleus (VMN) and hindbrain libraries described in Feng et al. [22]. The 2000 ng multiplexed cDNA pool was $2 \times 100$ paired-end sequenced on the Illumina HiSeq2000 in the Cornell University Institute of Biotechnology Genomics Facility.

\section{Transcriptome assembly and annotation}

The assembled and annotated transcriptome described here was the same transcriptome used for our companion study [22]. Illumina quality filtering was used to remove pairs of reads in which either of the paired reads was of poor quality. The Trimmomatic tool kit [72] was used to remove adaptor sequences and low quality nucleotides from the ends, and trimmed sequences of less than ten nucleotides were removed. Following filtering, there were $20.2 \pm 2.4$ million reads (mean $\pm \mathrm{SD}$ ) per tissue group remaining. These were transferred to the Pittsburgh Supercomputing Center's Blacklight system for de novo transcriptome assembly. Downstream analyses were performed with the Trinity version r2013-0215 software package on Cornell's Computational Biology Service Unit's computers [23, 24, 73]. In examining previously identified genes in our initial assembly, we found several problematic contigs in which, for example, paralogous steroid receptors were assembled into single gene components or assembled transcripts contained long extraneous sequences on one end, apparently representing portions of transcripts from other genes. The observation of these chimeras is likely due to the whole genome duplication in teleosts (see [74, 75]) and our interest in genes with multiple known orthologs (eg., estrogen, androgen, and glucocorticoid receptors). To reduce such chimeric assemblies, we employed the jaccard_clip function as well as set the min_kmer_cov at 2 . While these settings increased the likelihood of fragmentation of assembled transcripts, they substantially reduced the occurrence of chimeric transcripts in our final transcriptome and still maintained a final N50 value of 2647. These settings largely eliminated such chimeric assemblies among our closely examined transcripts. 
Following the initial assembly process, we filtered the transcriptome further to retain only transcripts that had an open reading frame (ORF) of at least 50 amino acids. While a 50 amino acid ORF is not stringent, we wanted to avoid eliminating potentially important short protein coding genes while reducing the number of non-coding genes. Though these non-coding genes may be critical to the variation in seasonal auditory physiology, their general lack of annotation makes such a bioinformatic analysis essentially impossible.

Using Trinity-supported downstream analysis tools, the initial HiSeq2000 reads were mapped back to the assembled transcriptome with Bowtie [76] in the RSEM [77] workflow to estimate abundances for each transcript and determine how well each assembled transcript was supported by the assigned reads. In cases of genes (components) with multiple transcripts (isoforms), if the number of reads that mapped to a given transcript (the IsoPct) was less than $1 \%$ of the total number of reads that mapped to all the transcripts for that gene, we considered that transcript to be lowly supported and eliminated it from our final transcriptome assembly.

After assembling the reads and filtering out poorly supported transcripts, we annotated the full transcriptome based on similarity to sequences in the NCBI nonredundant protein database using Blast2GO [78]. Within Blast2GO, we used blastx to compare each transcript to the NCBI protein database with an e-value cutoff of $10^{-10}$. We performed subsequent blastn analyses for sequences without significant blastx hits, though this added few annotation due to our previously excluding transcripts with ORFs of less than 50 amino acids. We then used Blast2GO for mapping and annotation of the transcripts as well as performing InterProScan and GO-Enzyme Code assignments.

\section{Analysis of most abundant transcripts}

We first performed analysis on the most highly expressed transcripts from reproductive and non-reproductive SE, regardless whether they were differentially expressed. In conjunction with analyses performed on differentially expressed transcripts below, this is useful for identifying reproductivestate dependent changes occurring at the level of the ear. Following Blast2GO annotation we identified the top 10 most abundant annotated transcripts within the reproductive and non-reproductive SE. We also mapped transcripts from reproductive and non-reproductive SE to KEGG functional pathways [79] using Blast2GO. The KEGG pathways were ranked in order of the number of transcripts assigned to each pathway in reproductive and non-reproductive SE, and the top 10 KEGG pathways were identified for each reproductive morph. This approach allowed us to compare the relative level of transcription dedicated to different pathways in the SE across reproductive states.

\section{Analysis of differentially expressed transcripts}

Differential expression analyses were performed within Trinity using RSEM and edgeR [80] following Trinity's standard differential analysis protocol. Though we focused largely on differential transcript expression, we performed these differential expression analyses on both the genes and the transcripts to allow comparisons between regulation at the gene and isoform levels. We used edgeR to estimate the common dispersion using a subset of 228 core eukaryotic genes (CEGs) $[89,90]$ selected based on having little or no direct involvement in cellular respiration, transcription, or translation. Initial analyses indicated that transcripts with these functions are highly differentially expressed across reproductive states in the ears, and thus would likely give an inaccurate dispersion estimate. The calculated dispersion value of 0.13317 was used in determining differential expression. We used a minimum 4-fold abundance difference with a maximum false discovery rate (FDR) of 0.001 as our criteria for selecting differentially expressed genes and transcripts. We used one-tailed Fisher's exact tests within Blast2GO with a maximum FDR of 0.05 to look for GO term enrichment among the seasonally differentially expressed transcripts relative to the whole suite of transcripts expressed in the ears across both seasons. As described above, assignment of transcripts to KEGG [79] functional pathways was performed based on Blast2GO annotation and used to examine differential expression of these pathways across reproductive states within the SE.

In addition to the differential analysis across the whole transcriptome, we isolated, in silico, the normalized read counts of two subsets of transcripts: those that had been annotated as either ion channels or steroid-related. We then performed differential analyses on these subsets with the same methods and parameters used for the whole transcriptome (ie, 4-fold differential abundance and FDR $<$ 0.001). We performed this targeted approach to allow for fewer comparisons and thus a less stringent FDR correction. We selected these particular subsets of transcripts based on previous studies that have implicated steroid hormones and the large conductance, calcium-activated potassium (BK) channel in seasonal variation in auditory sensitivity $[12,13]$. This facilitated the identification of more differentially expressed candidate transcripts with important functional implications.

\section{Availability of supporting data}

The final assembled transcriptome and reads from each sample group have been submitted to the NCBI Transcriptome Shotgun Assembly and Sequence Read Archive databases under BioProject accession number [PRJNA269550]. 


\section{Additional files}

Additional file 1: Differentially expressed steroid-related transcripts in saccular epithelium (SE). Seasonal differential analysis in the SE was performed with the subset of steroid-related transcripts. Top hit BLAST hit descriptions for each transcript are shown. (DOCX $16 \mathrm{~kb}$ )

Additional file 2: Differentially expressed ion channel transcripts in saccular epithelium (SE). Seasonal differential analysis in the SE was performed with the subset of ion channel transcripts. Top hit BLAST hit descriptions for each transcript are shown. (DOCX $18 \mathrm{~kb}$ )

\section{Abbreviations}

BK: Large conductance, calcium-activated potassium; BLAST: Basic local alignment search tool; ERR: Estrogen related receptor; FDR: False discovery rate; FPKM: Fragments per kilobase of transcript per million reads mapped; GO: Gene ontology; KEGG: Kyoto encyclopedia of genes and genomes; SE: Saccular epithelium; VMN: vocal motor nucleus; 11KT: 11-ketotestosterone.

\section{Competing interests}

The authors declare that they have no competing interests.

\section{Authors' contributions}

DJF, NYF and AHB designed the experiments; DJF and NYF conducted the experiments; DJF, NYF and AHB analyzed data and wrote the paper. All authors read and approved the final manuscript.

\section{Authors' information}

Not applicable

\section{Availability of data and materials}

Not applicable

\section{Acknowledgements}

We thank the Jim Giovannoni lab (USDA-ARS, Cornell University), especially Silin Zhong for help with cDNA library construction. We are grateful to Kevin Rohmann for providing us with the SE samples used for these analyses. Cornell University BioHPC Lab resources and the Bioinformatics Facility provided us with computing resources and expertise to complete our project. We are grateful to the Broad Institute, particularly Brian Haas, for providing and assisting with the Trinity software package. This work used the Extreme Science and Engineering Discovery Environment (XSEDE) supported by National Science Foundation grant OCl-1053575. This research was supported by NSF IOS 1120925 (AHB) and Cornell University's Center for Vertebrate Genomics (NYF).

\section{Author details}

${ }^{1}$ Department of Neurobiology and Behavior, Cornell University, Ithaca, NY 14853, USA. ${ }^{2}$ Current Address: North Carolina Museum of Natural Sciences, Genomics and Microbiology, Raleigh, NC 27601, USA.

Received: 6 May 2015 Accepted: 19 September 2015

Published online: 14 October 2015

\section{References}

1. Hardin PE, Hall JC, Rosbash M. Feedback of the Drosophila period gene product on circadian cycling of its messenger RNA levels. Nature. 1990;343(6258):536-40.

2. Glossop NRJ, Lyons LC, Hardin P. Interlocked feedback loops within the Drosophila circadian oscillator. Science. 1999;286(5440):766-8.

3. Collins B, Mazzoni EO, Stanewsky R, Blau J. Drosophila CRYPTOCHROME is a circadian transcriptional repressor. Curr Biol. 2006;16(5):441-9.

4. Zheng B, Larkin DW, Albrecht U, Sun ZS, Sage M, Eichele G, et al. The mPer2 gene encodes a functional component of the mammalian circadian clock. Nature. 1999;400(6740):169-73.

5. Chandrasekaran S, Ament SA, Eddy JA, Rodriguez-Zas SL, Schatz BR, Price ND, et al. Behavior-specific changes in transcriptional modules lead to distinct and predictable neurogenomic states. Proc Natl Acad Sci U S A. 2011;108(44):18020-5.
6. Whitfield CW, Cziko A-M, Robinson GE. Gene expression profiles in the brain predict behavior in individual honey bees. Science. 2003;302(5643):296-9.

7. Mello CV, Clayton DF. The opportunities and challenges of large-scale molecular approaches to songbird neurobiology. Neurosci Biobehav Rev. 2014;50:70-6.

8. McKibben JR, Bass AH. Behavioral assessment of acoustic parameters relevant to signal recognition and preference in a vocal fish. J Acoust Soc Am. 1998;104(6):3520-33.

9. Sisneros JA, Bass AH. Seasonal plasticity of peripheral auditory frequency sensitivity. J Neurosci. 2003;23(3):1049-58.

10. Sisneros JA. Seasonal plasticity of auditory saccular sensitivity in the vocal plainfin midshipman fish, Porichthys notatus. J Neurophysiol. 2009;102(2):1121-31.

11. Rohmann $\mathrm{KN}$, Bass $\mathrm{AH}$. Seasonal plasticity of auditory hair cell frequency sensitivity correlates with plasma steroid levels in vocal fish. J Exp Biol. 2011;214(11):1931-42.

12. Rohmann KN, Fergus DJ, Bass AH. Plasticity in ion channel expression underlies variation in hearing during reproductive cycles. Curr Biol. 2013:23(8):678-83.

13. Sisneros JA, Forlano PM, Deitcher DL, Bass AH. Steroid-dependent auditory plasticity leads to adaptive coupling of sender and receiver. Science. 2004;305(5682):404-7.

14. Kilicdag EB, Yavuz H, Bagis T, Tarim E, Erkan AN, Kazanci F. Effects of estrogen therapy on hearing in postmenopausal women. Am J Obstet Gynecol. 2004;190(1):77-82.

15. Cohen MJ, Winn HE. Electrophysiological observations on hearing and sound production in the fish, Porichthys notatus. J Exp Zool. 1967;165(3):355-69.

16. Fergus DJ, Bass AH. Localization and divergent profiles of estrogen receptors and aromatase in the vocal and auditory networks of a fish with alternative mating tactics. J Comp Neurol. 2013:521(12):2850-69.

17. Forlano PM, Deitcher DL, Bass AH. Distribution of estrogen receptor alpha mRNA in the brain and inner ear of a vocal fish with comparisons to sites of aromatase expression. J Comp Neurol. 2005;483(1):91-113.

18. Fergus DJ, Feng NY, Bass AH. Gene expression patterns in auditory hair cell epithelium: Insights into the molecular basis of seasonal plasticity in frequency sensitivity. Washington DC: Society for Neuroscience Annual Meeting; 2014. 183.02/SS28.

19. Brantley R, Bass AH. Alternative male spawning tactics and acoustic-signals in the plainfin midshipman fish Porichthys notatus girard (Teleostei, Batrachoididae). Ethology. 1994;96(3):213-32.

20. Mclver EL, Marchaterre MA, Rice AN, Bass AH. Novel underwater soundscape: acoustic repertoire of plainfin midshipman fish. J Exp Biol. 2014;217:2377-89.

21. Whitchurch EA, Sisneros JA. Seasonal plasticity of saccular sensitivity in the type II sneaker-male plainfin midshipman fish (Porichthys notatus). J Acoust Soc Am. 2011;129(4):2471.

22. Feng NY, Fergus DJ, Bass AH. Neural transcriptome reveals molecular mechanisms for temporal control of vocalization across multiple timescales. BMC Genomics. 2015;16(1):408.

23. Grabherr MG, Haas BJ, Yassour M, Levin JZ, Thompson DA, Amit I, et al. Full-length transcriptome assembly from RNA-Seq data without a reference genome. Nat Biotechnol. 2011;29(7):644-52.

24. Haas BJ, Papanicolaou A, Yassour M, Grabherr M, Blood PD, Bowden J, et al. De novo transcript sequence reconstruction from RNA-seq using the Trinity platform for reference generation and analysis. Nat Protoc. 2013:8(8):1494-512.

25. Kandel ER, Schwartz JH, Jessell TM, Siegelbaum SA. Hudspeth AJ. Fifth Edition. McGraw-Hill Professional: Principles of Neural Science; 2013.

26. Li F, Zhang D, Fujise K. Characterization of fortilin, a novel antiapoptotic protein. J Biol Chem. 2001;276(50):47542-9.

27. Gnanasekar M, Thirugnanam S, Zheng G, Chen A, Ramaswamy K. Gene silencing of translationally controlled tumor protein (TCTP) by siRNA inhibits cell growth and induces apoptosis of human prostate cancer cells. Int J Oncol. 2009;34(5):1241-6.

28. Coffin AB, Mohr RA, Sisneros JA. Saccular-specific hair cell addition correlates with reproductive state-dependent changes in the auditory saccular sensitivity of a vocal fish. J Neurosci. 2012:32(4):1366-76.

29. Scheffer DI, Shen J, Corey DP, Chen Z-Y. Gene expression by mouse inner ear hair cells during development. J Neurosci. 2015;35(16):6366-80.

30. Chen S, Zhou D, Yang C, Sherman M. Molecular basis for the constitutive activity of estrogen-related receptor alpha-1. J Biol Chem. 2001;276(30):28465-70. 
31. Zhang Z, Teng CT. Estrogen receptor-related receptor interacts with coactivator and constitutively activates the estrogen response elements of the human lactoferrin gene. J Biol Chem. 2000;275(27):20837-46.

32. Xie W, Hong H, Yang NN, Lin RJ, Simon CM, Stallcup MR, et al. Constitutive activation of transcription and binding of coactivator by estrogen-related receptors 1 and 2. Mol Endocrinol. 1999;13(12):2151-62.

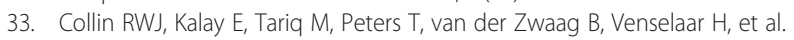
Mutations of ESRRB encoding estrogen-related receptor beta cause autosomal-recessive nonsyndromic hearing impairment DFNB35. Am J Hum Genet. 2008;82(1):125-38.

34. Lee K, Khan S, Ansar M, Santos-Cortez RLP, Ahmad W, Leal SM. A novel ESRRB deletion is a rare cause of autosomal recessive nonsyndromic hearing impairment among Pakistani families. Genet Res Int. 2011;2011:4.

35. Nolan LS, Maier H, Hermans-Borgmeyer I, Girotto G, Ecob R, Pirastu N, et al. Estrogen-related receptor gamma and hearing function: evidence of a role in humans and mice. Neurobiol Aging. 2013;34(8):2077.

36. Drescher DG, Ramakrishnan N a, Drescher MJ, Chun W, Wang X, Myers SF, et al. Cloning and characterization of alpha9 subunits of the nicotinic acetylcholine receptor expressed by saccular hair cells of the rainbow trout (Oncorhynchus mykiss). Neuroscience. 2004;127(3):737-52.

37. Chagnaud BP, Bass AH. Vocal corollary discharge communicates call duration to vertebrate auditory system. J Neurosci. 2013;33(48):18775-80.

38. Elgoyhen $A B$, Katz E. The efferent medial olivocochlear-hair cell synapse. J Physiol Paris. 2012;106(1-2):47-56.

39. Bass A, Clark $C$. The physical acoustics of underwater sound communication. In: Simmons A, Fay R, Popper A, editors. Acoustic Communication SE - 2, vol. Volume 16. New York: Springer; 2003. p. 15-64.

40. Urick RJ. Principles of Underwater Sound. 2nd edition. New York: McGraw-Hill; 1975

41. Huang M, Kantardzhieva A, Scheffer D, Liberman MC, Chen Z-Y. Hair cell overexpression of Islet1 reduces age-related and noise-induced hearing loss. J Neurosci. 2013;33(38):15086-94.

42. Lynch ED, Lee MK, Morrow JE, Welcsh PL, León PE, King MC. Nonsyndromic deafness DFNA1 associated with mutation of a human homolog of the Drosophila gene diaphanous. Science. 1997;278(5341):1315-8.

43. Watanabe N. p140mDia, a mammalian homolog of Drosophila diaphanous, is a target protein for Rho small GTPase and is a ligand for profilin. EMBO J. 1997;16(11):3044-56.

44. Wallar BJ, Alberts AS. The formins: active scaffolds that remodel the cytoskeleton. Trends Cell Biol. 2003;13(8):435-46.

45. Schoen CJ, Emery SB, Thorne MC, Ammana HR, Sliwerska E, Arnett J, et al. Increased activity of Diaphanous homolog 3 (DIAPH3)/diaphanous causes hearing defects in humans with auditory neuropathy and in Drosophila. Proc Natl Acad Sci U S A. 2010;107(30):13396-401.

46. Schuck JB, Sun H, Penberthy WT, Cooper NGF, Li X, Smith ME. Transcriptomic analysis of the zebrafish inner ear points to growth hormone mediated regeneration following acoustic trauma. BMC Neurosci. 2011;12:88.

47. Kusakabe M, Nakamura I, Young G. 11 beta-hydroxysteroid dehydrogenase complementary deoxyribonucleic acid in rainbow trout: cloning, sites of expression, and seasonal changes in gonads. Endocrinology. 2003;144(6):2534-45.

48. Bury NR, Sturm A. Evolution of the corticosteroid receptor signalling pathway in fish. Gen Comp Endocrinol. 2007;153(1-3):47-56.

49. Rarey KE, Curtis LM. Receptors for glucocorticoids in the human inner ear. Otolaryngol Neck Surg. 1996;115(1):38-41.

50. Henkin Rl, Daly RL. Auditory detection and perception in normal man and in patients with adrenal cortical insufficiency: effect of adrenal cortical steroids. J Clin Invest. 1968;47(6):1269-80.

51. Siaud P, Maurel D, Lucciano M, Kosa E, Cazals Y. Enhanced cochlear acoustic sensitivity and susceptibility to endotoxin are induced by adrenalectomy and reversed by corticosterone supplementation in rat. Eur J Neurosci. 2006;24(12):3365-71.

52. Beckwith BE, Lerud K, Antes JR, Reynolds BW. Hydrocortisone reduces auditory sensitivity at high tonal frequencies in adult males. Pharmacol Biochem Behav. 1983;19(3):431-3.

53. Simoens VL, Hébert $\mathrm{S}$. Cortisol suppression and hearing thresholds in tinnitus after low-dose dexamethasone challenge. BMC Ear, Nose Throat Disord. 2012;12:4.

54. Namdaran P, Reinhart KE, Owens KN, Raible DW, Rubel EW. Identification of modulators of hair cell regeneration in the zebrafish lateral line. J Neurosci. 2012;32(10):3516-28
55. Brantley RK, Wingfield JC, Bass AH. Sex steroid levels in Porichthys notatus, a fish with alternative reproductive tactics, and a review of the hormonal bases for male dimorphism among teleost fishes. Horm Behav. 1993;27(3):332-47.

56. Sisneros JA, Forlano PM, Knapp R, Bass AH. Seasonal variation of steroid hormone levels in an intertidal-nesting fish, the vocal plainfin midshipman. Gen Comp Endocrinol. 2004;136(1):101-16.

57. Remage-Healey L, Bass AH. Rapid, hierarchical modulation of vocal patterning by steroid hormones. J Neurosci. 2004;24(26):5892-900.

58. Remage-Healey L, Bass AH. Plasticity in brain sexuality is revealed by the rapid actions of steroid hormones. J Neurosci. 2007;27(5):1114-22.

59. Forlano PM, Marchaterre M, Deitcher DL, Bass AH. Distribution of androgen receptor mRNA expression in vocal, auditory, and neuroendocrine circuits in a teleost fish. J Comp Neurol. 2010;518(4):493-512.

60. Maruska KP, Fernald RD. Steroid receptor expression in the fish inner ear varies with sex, social status, and reproductive state. BMC Neurosci. 2010;11:58.

61. Rohmann KN, Tripp J a, Genova RM, Bass AH. Manipulation of BK channel expression is sufficient to alter auditory hair cell thresholds in larval zebrafish. J Exp Biol. 2014;217(14):2531-9.

62. Fettiplace R, Fuchs PA. Mechanisms of hair cell tuning. Annu Rev Physiol. 1999;61:809-34.

63. Bennett MVL, Zukin RS. Electrical coupling and neuronal synchronization in the Mammalian brain. Neuron. 2004;41(4):495-511.

64. Chagnaud BP, Zee MC, Baker R, Bass AH. Innovations in motoneuron synchrony drive rapid temporal modulations in vertebrate acoustic signaling. J Neurophysiol. 2012;107(12):3528-42.

65. Teubner B, Michel V, Pesch J, Lautermann J, Cohen-Salmon M, Söhl G, et al. Connexin30 (Gjb6)-deficiency causes severe hearing impairment and lack of endocochlear potential. Hum Mol Genet. 2003;12(1):13-21.

66. Sokolowski BH, Popper AN. Transmission electron microscopic study of the saccule in the embryonic, larval, and adult toadfish Opsanus tau. J Morphol. 1988;198(1):49-69.

67. Hirono M, Ogawa Y, Misono K, Zollinger DR, Trimmer JS, Rasband MN, et al. BK channels localize to the paranodal junction and regulate action potentials in myelinated axons of cerebellar purkinje cells. J Neurosci. 2015;35(18):7082-94.

68. McKibben JR, Bass AH. Peripheral encoding of behaviorally relevant acoustic signals in a vocal fish: single tones. J Comp Physiol A. 1999;184(6):563-76.

69. Bass AH. Neural mechanisms of vocal communication: Interfacing with neuroendocrine mechanisms. In Behaviour and Neurodynamics for Auditory Communication. Edited by Kanwal JS, Ehret G. New York: Cambridge University Press; 2006:123-131.

70. Vasconcelos RO, Sisneros JA, Amorim MCP, Fonseca PJ. Auditory saccular sensitivity of the vocal Lusitanian toadfish: low frequency tuning allows acoustic communication throughout the year. J Comp Physiol A Neuroethol Sens Neural Behav Physiol. 2011;197(9):903-13.

71. Zhong S, Joung JG, Zheng Y, Chen YR, Liu B, Shao Y, et al. High-throughput illumina strand-specific RNA sequencing library preparation. Cold Spring Harb Protoc. 2011;2011(8):940-9.

72. Bolger AM, Lohse M, Usadel B. Trimmomatic: A flexible trimmer for Illumina sequence data. Bioinformatics. 2014;30(15):2114-20.

73. Li B, Fillmore N, Bai Y, Collins M, Thomson JA, Stewart R, et al. Evaluation of de novo transcriptome assemblies from RNA-Seq data. Genome Biol. 2014;15:553.

74. Taylor JS, Braasch I, Frickey T, Meyer A, Van de Peer Y. Genome duplication, a trait shared by 22000 species of ray-finned fish. Genome Res. 2003;13(3):382-90.

75. Glasauer SMK, Neuhauss SCF. Whole-genome duplication in teleost fishes and its evolutionary consequences. Mol Genet Genomics. 2014;289(6):1045-60

76. Langmead B, Trapnell C, Pop M, Salzberg SL. Ultrafast and memory-efficient alignment of short DNA sequences to the human genome. Genome Biol. 2009;10(3):R25

77. Li B, Dewey CN. RSEM: accurate transcript quantification from RNA-Seq data with or without a reference genome. BMC Bioinformatics. 2011;12(1):323.

78. Götz S, García-Gómez JM, Terol J, Williams TD, Nagaraj SH, Nueda MJ, et al. High-throughput functional annotation and data mining with the Blast2GO suite. Nucleic Acids Res. 2008;36(10):3420-35.

79. Kanehisa M, Goto S. KEGG: Kyoto encyclopaedia of genes and genomes. Nucl Acids Res. 2000;28(1):27-30. 
80. Robinson MD, McCarthy DJ, Smyth GK. edgeR: a Bioconductor package for differential expression analysis of digital gene expression data. Bioinformatics. 2010;26(1):139-40.

81. Seal RP, Akil O, Yi E, Weber CM, Grant L, Yoo J, et al. Sensorineural deafness and seizures in mice lacking vesicular glutamate transporter 3. Neuron 2008;57(2):263-75.

82. $\mathrm{Ng}$ L, Rüsch A, Amma LL, Nordström K, Erway LC, Vennström B, et al. Suppression of the deafness and thyroid dysfunction in Thrb-null mice by an independent mutation in the Thra thyroid hormone receptor alpha gene. Hum Mol Genet. 2001;10(23):2701-8.

83. Kim AH, Nahm E, Sollas A, Mattiace L, Rozental R. Connexin 43 and hearing: Possible implications for retrocochlear auditory processing. Laryngoscope. 2013;123(12):3185-93.

84. Lee SI, Conrad T, Jones SM, Lagziel A, Starost MF, Belyantseva IA, et al. A null mutation of mouse Kcna10 causes significant vestibular and mild hearing dysfunction. Hear Res. 2013;300:1-9.

85. Mackenzie FE, Parker A, Parkinson NJ, Oliver PL, Brooker D, Underhill P, et al. a, Lukashkin a N, Holmes C, Brown SDM. Analysis of the mouse mutant Cloth-ears shows a role for the voltage-gated sodium channel Scn8a in peripheral neural hearing loss. Genes Brain Behav. 2009;8(7):699-713.

86. Rubinato E, Morgan A, D'Eustacchio A, Pecile V, Gortani G, Gasparini P, et al. A novel deletion mutation involving TMEM38B in a patient with autosomal recessive osteogenesis imperfecta. Gene. 2014;545(2):290-2.

87. Pirone A, Kurt S, Zuccotti A, Rüttiger L, Pilz P, Brown DH, et al. a2 $\delta 3$ is essential for normal structure and function of auditory nerve synapses and is a novel candidate for auditory processing disorders. J Neurosci. 2014;34(2):434-45.

88. Segil N, Tao L. Early transcriptional response to aminoglycoside antibiotic suggests alternate pathways leading to apoptosis of sensory hair cells in the mouse inner ear. Front Cell Neurosci. 2015;9:190.

89. Parra G, Bradnam K, Korf I. CEGMA: a pipeline to accurately annotate core genes in eukaryotic genomes. Bioinformatics. 2007; 23:1061-7.

90. Parra G, Bradnam K, Ning Z, Keane T, Korf I. Assessing the gene space in draft genomes. Nucleic Acids Res. 2009; 37:289-97.

\section{Submit your next manuscript to BioMed Central and take full advantage of:}

- Convenient online submission

- Thorough peer review

- No space constraints or color figure charges

- Immediate publication on acceptance

- Inclusion in PubMed, CAS, Scopus and Google Scholar

- Research which is freely available for redistribution 